\title{
Channel Modeling Based on Interference Temperature in Underlay Cognitive Wireless Networks
}

\author{
Manuj Sharma ${ }^{\# 1}$, Anirudha Sahoo ${ }^{\# 2}$, K. D. Nayak ${ }^{*}$ \\ \# Dept. of Computer Science \& Engineering \\ Indian Institute of Technology Bombay, Mumbai, India \\ manuj@it.iitb.ac.in \\ sahoo@cse.iitb.ac.in \\ * Advanced Numerical Research \& Analysis Group \\ Defence Research \& Development Organization, Hyderabad, India
}

\begin{abstract}
Cognitive radio based dynamic spectrum access network is emerging as a technology to address spectrum scarcity. In this study, we assume that the channel is licensed to some primary (licensed) operator. We consider a sensor network with cognitive radio capability that acts as a secondary (unlicensed) network and uses the channel in underlay mode. The secondary network uses interference temperature model [1] to ensure that the interference to the primary devices remain below a predefined threshold. We use Hidden Markov Model (HMM) to model the interference temperature dynamics of a primary channel. The HMM is trained using Baum-Welch procedure. The trained HMM is shown to be statistically stable. Secondary nodes use this trained HMM to predict the interference temperature of the channel in future time slots and computes the value of Channel Availability Metric (CAM) for the channel. CAM is used by secondary nodes to select a primary channel for transmission. Results of application of such trained HMMs in channel selection in multi-channel wireless network are presented.
\end{abstract}

\section{INTRODUCTION}

Cognitive radio based Dynamic Spectrum Access (DSA) networks have emerged as a promising way to counter the challenges of electromagnetic spectrum scarcity, which has primarily resulted from underutilization of licensed spectrum bands. In this paper, we consider a cognitive radio-enabled sensor network, which acts as an unlicensed secondary network and uses a licensed channel in underlay mode. The channel is assumed to be licensed to some primary operator. The sensor nodes follow the interference temperature model [1]. Interference temperature is defined as a measure of the $\mathrm{RF}$ power available at a receiving antenna to be delivered to a receiver. This is the power generated by other emitters and noise sources. More specifically, it is the temperature equivalent of the RF power available at a receiving antenna per unit of bandwidth, measured in units of Kelvin [1,2]. If $f_{c}$ and $B_{c}$ denote the central frequency and bandwidth (in Hertz) of channel $c, P_{i}\left(f_{c}, B_{c}\right)$ denote the power in Watt (at the antenna of a receiving or measuring device), and $k$ denote the Boltzmann's constant $\left(1.38 \times 10^{-23}\right.$ Joules per Kelvin), then the interference temperature for channel $c$ is given by [2]:

$$
T_{c}\left(f_{c}, B_{c}\right)=\frac{P_{i}\left(f_{c}, B_{c}\right)}{k B_{c}}
$$

In interference temperature model, a secondary device senses the available spectrum band (also termed as channel in this paper) to compute the aggregate interference and its equivalent temperature experienced at each channel. It then transmits the data on a channel provided the interference temperature for that channel does not exceed a predefined threshold at any primary receiver within the transmitter's interference range. The secondary device usually transmit with very low-power and follow an underlay model for accessing licensed spectrum.

We model the interference temperature dynamics of a channel, which is used by a cognitive sensor network, using a hidden Markov model (HMM). The HMM is trained with the observed interference temperature values using Baum-Welch procedure [3]. The trained model is then validated and used as a sequence generator to predict the interference temperature dynamics on the channel in the future. The prediction is used to select a preferable channel for communication.

\section{RELATED WORK}

Hidden Markov Models have been used in the area of speech recognition [3], characterization of errors in digital radio channels [4], and statistical analysis of an Internet communication channel [5]. In the context of dynamic spectrum access networks, HMMs are used to model the occupancy of a licensed band by licensed user, and the resulting throughput available to the secondary devices based on this occupancy [6]. HMM has also been used in detection and classification of very low SNR signals in cognitive radio [7]. A non-binary, receiver-centric constraint model for channel assignment is proposed in [8], which allows for assignment of same channel to more than one transmitter provided the interference temperature at any receiver does not exceed the threshold.

\section{PROBLEM DEFINITION}

We consider a wireless sensor network in which each node is assumed to contain cognitive radio modules that can 
periodically scan and sense multiple licensed channels to compute the interference temperature at each channel. It then compares the computed interference temperature value (at each channel) with a predefined threshold value. We consider a channel $c$. Let us assume that the interference temperature threshold for channel $c$ is denoted as $I T_{t h}$, and the interference temperature measured by a node for this channel at any given observation instant $t$ is denoted as $I T_{t}$. At each instant $t$, the node records an observation symbol $\left(O_{t}\right)$ depending upon the following conditions:

$$
O_{t}=0 \text { if } I T_{t} \leq I T_{t h} ; O_{t}=1 \text { if } I T_{t}>I T_{t h}
$$

The node periodically makes such observations, and records an observation sequence $O=O_{1} \ldots O_{T}$, (where $O_{t} \in$ $[0,1] \forall t=1 \ldots \mathrm{T})$ over a period of, say $\mathrm{T}$ time slots. We represent such observation sequence as $O=\left\{O_{t}\right\}_{t=l}^{T}$. The more the number of 1 's in the observation sequence, the higher the interference indicated for the channel at the node. The specific problem addressed in this paper is as follows:

1. Given an observation sequence for first $T$ time slots for channel c,

$$
O=\left\{O_{t}\right\}_{t=1}^{T},
$$

can the node predict the observation sequence for next T time slots for the same channel

$$
O_{\text {next }}=\left\{O_{t}\right\}_{t=T+1}^{2 T} ?
$$

2. Can such a prediction be used by the node for selecting preferable channel for communication?

\section{THE Proposed HidDEN MARKov MOdeL}

In order to solve the above mentioned problem, we model a channel as a hidden Markov chain, and train this model using an observation sequence $O$, called as training observation sequence. Here, $O$ is a sequence of symbols 0 and $1 \mathrm{~s}$, as defined in the previous section. The trained HMM is then checked for statistical stability and accuracy using a number of previously unseen test observation sequences, obtained for the same channel. Once a credible trained HMM for a channel is obtained, we use the sequences generated by the HMM to compute a channel availability metric. A channel having higher availability metric value is selected by the node as preferable channel for communication.

In order to train and test an HMM, we obtain an observation sequence by simulating a secondary sensor network deployed as a regular mesh and make the interference temperature measurements at one of the designated sensor nodes in the simulated environment. Each measured interference temperature value is compared against a predefined threshold and an observation symbol 0 or 1 is recorded according to (2). In this manner, over a period of T time slots, we obtain the observation sequence $O=O_{1} \ldots O_{T}$ A hidden Markov chain is trained with the obtained training sequence $O$ using Baum Welch procedure [3]. Baum-Welch procedure is an Expectation-Maximization (EM) procedure for HMM (for details, please see [3]). The trained model is used to predict the future channel behavior with respect to interference temperature. It is represented as $\lambda_{m}=(A, B, \pi)$, along with symbols $\mathrm{N}$ and $\mathrm{M}$, where $\mathrm{N}$ is the number of states in the model, $\mathrm{M}$ is the total number of distinct observation symbols, $A$ is the state transition probability matrix, $B$ is the observation symbol probability distribution matrix, and $\pi$ is the initial state probability distribution vector. In our model, $\mathrm{M}=2$ (the observation symbols are 0 and 1 ).

In order to validate the trained HMM on unseen observation sequences, we obtain a set of other observation sequences $O_{i}^{\prime}(1 \leq \mathrm{i} \leq \Gamma$, where $\Gamma$ is the cardinality of the set), after obtaining sequence $O$, through simulation of the same model. These sequences are called as the test sequences. We compute the log-likelihood $\left(\ell_{i}\right)$ of occurrence of each previously unseen test sequence $O_{i}$ using the trained HMM $\lambda_{m}$. The statistical stability of the trained HMM is validated by plotting the value $\ell_{i}$ of for each test sequence $i$ (where $1 \leq i \leq$ $\Gamma$ ), which should have as little variation as possible (the lesser the variation, more stable is the model). The log-likelihood values for these test sequences should be higher when computed using trained model in comparison with untrained model.

\section{ApPlication of TRAined HMm: ChanNEL SELECTION IN MULTI-CHANNEL WIRELESS NETWORK}

One potential application of HMM that we investigate and propose in this paper is to use the sequences generated by the trained HMM in selecting preferable channel for communication in multi-channel cognitive wireless network. Consider a multi-channel cognitive wireless network with $n$ channels. Let us assume that the training sequences for these channels is obtained by a designated sensor node, which construct hidden Markov model for each channel using these training sequences, as explained earlier. Let $\mathrm{H}_{\mathrm{i}}$ represent the trained HMM for channel i, $\mathrm{GS}_{\mathrm{Hi}}$ denote the binary sequence generated by $\mathrm{H}_{\mathrm{i}},\left|\mathrm{GS}_{\mathrm{Hi}}\right|$ denote the length of sequence $\mathrm{GS}_{\mathrm{Hi}}$, $\mathrm{GS}_{\mathrm{Hi}}^{1}$ denote the number of 1 's in the generated sequence $\mathrm{GS}_{\mathrm{Hi}}$, and $\beta_{\mathrm{i}}$ denote the average gap between any two 1's in the generated sequence $\mathrm{GS}_{\mathrm{Hi}}$. Then, we define channel availability metric $\left(\mathrm{CAM}_{\mathrm{i}}\right)$ for channel $\mathrm{i}$ as follows:

$$
\mathrm{CAM}_{\mathrm{i}}=\beta_{\mathrm{i}}+\frac{1}{\left(\mathrm{GS}_{\mathrm{Hi}}^{1} /\left|\mathrm{GS}_{\mathrm{Hi}}\right|\right)}
$$

The channel availability metric is higher for a channel for which the trained HMM predicts lower number of slots with symbol 1 (i.e., lesser number of slots in which interference temperature exceeds the threshold), and larger separations (i.e. higher spread) between symbol $1 \mathrm{~s}$ in the generated sequence. The node selects the channel with highest value of channel availability metric $\left(\mathrm{CAM}_{\mathrm{i}}\right)$, as the most preferable channel for communication. As an example, consider a sequence $\mathrm{GS}_{\mathrm{Hi}}=$ 0100101100011, generated by the trained HMM for the ith channel. For this sequence, the gap between symbol 1 
appearing at position 2 and 5 is equal to 2 , between positions 5 and 7 is 1 , between positions 7 and 8 is 0 , between positions 8 and 12 is 3 , and between positions 12 and 13 is 0 . The average gap between any two 1's in this generated sequence is equal to, $\beta_{\mathrm{i}}=(2+1+0+3+0) / 5=1.2$. For this sequence, $\mathrm{GS}_{\mathrm{Hi}}^{1}=6$ and $\left|\mathrm{GS}_{\mathrm{Hi}}\right|=13$. Therefore, by (3), $\mathrm{CAM}_{\mathrm{i}}=3.37$.

\section{SIMULATION RESULTS}

\section{A. Simulated Network Model for Obtaining Network Sequences}

We simulate a sensor network of 30 stationary sensor nodes deployed as regular mesh of 6 rows x 5 columns, and placed in an area of $200 \times 250$ meters using OPNET simulator [9]. We simulate a licensed channel that roughly correspond to one GSM channel having a carrier frequency of $900 \mathrm{MHz}$, bandwidth of $200 \mathrm{KHz}$, and supported data rate of $270 \mathrm{Kbps}$. The channel is assumed to be licensed to some primary operator. The sensor network acts as a secondary network, which dynamically uses the licensed channel in underlay mode. In simulations, we assume that the time is slotted and each slot is equal to the time required to transmit a fixed-size information packet. Transmissions by sensor nodes on channel $c$ contribute to the interference generated by the secondary sensor network. We focus on one of the designated sensor nodes (referred to as measurement node), which performs the measurement and computation of interference temperature of the designated channel $c$. Similar computations should be done for all the other available channels on all the sensor nodes. The interference threshold for channel $c$ is assumed to be equal to $10^{-8} \mathrm{~W}$, which is roughly equal to the interference temperature of $362 \times 10^{7} \mathrm{~K}$. In each time slot, if the measurement node senses interference and computes the equivalent temperature to be exceeding the above threshold value, then it records symbol 1; otherwise, it records symbol 0. A total of $\mathrm{L}$ observation symbols are generated, which are divided into ( $\mathrm{L} / \mathrm{T}$ ) subsequences, each of length $\mathrm{T}$. The first subsequence is used as training sequence, whereas the remaining are used as test sequences. After obtaining all the training and test observation sequences, the node then constructs and trains 2-state, 3-state and 4-state HMMs using the training sequences and Baum-Welch procedure. It then validates the constructed HMMs using the test sequences. The transmission parameters of the sensor nodes and measurement node are same and are given in Table I. HMM related parameters are given in Table II.

We assume that the traffic model for sensing application at each sensor node corresponds to ON-OFF traffic model, where ON period for a node corresponds to the period for which it senses the event of interest and transmit information packets towards a designated monitoring station, and OFF period corresponds to the period in which it does not senses any event. The patches of the region where events occur more frequently and for longer durations are referred to as red patches, whereas the patches where events occur relatively less frequently and for shorter durations are referred to as green patches. A set of sensor nodes are randomly selected to
TABLE I

TRANSMISSION PARAMETERS FOR SENSOR NODES

\begin{tabular}{|c|c|}
\hline Parameters & Value \\
\hline Carrier Frequency & $900 \mathrm{MHz}$ \\
\hline Bandwidth & $200 \mathrm{KHz}$ \\
\hline Channel data rate & $270 \mathrm{Kbps}$ \\
\hline Modulation & bpsk \\
\hline Transmission power & 0.01 watts \\
\hline Packet size & 2700 bits \\
\hline Packet format & unformatted \\
\hline Traffic source model & $\begin{array}{c}\text { Exponentially distributed ON-OFF } \\
\text { model (Sensor node) }\end{array}$ \\
\hline Mean OFF sojorn time for & $\begin{array}{c}\text { For nodes belonging to red patches: } \\
10 \text { seconds }\end{array}$ \\
sensor nodes & $\begin{array}{c}\text { For nodes belonging to green patches: } \\
30 \text { seconds }\end{array}$ \\
& $\begin{array}{c}\text { For nodes belonging to red patches: } \\
30 \text { seconds }\end{array}$ \\
Mean ON sojorn time for sensor \\
nodes
\end{tabular}

TABLE II

HidDEN MARKOV MODEL PARAMETERS

\begin{tabular}{|c|c|}
\hline Parameter & Value \\
\hline No. of states $(\mathrm{N})$ & $2,3,4$ \\
\hline No. of distinct symbols (M) & 2 \\
\hline Symbol set (V) & $\{0,1\}$ \\
\hline Training sequence length & 300 symbols \\
\hline Testing sequence length & 300 symbols \\
\hline No. of training sequences & 1 \\
\hline No. of testing sequences & 6 \\
\hline
\end{tabular}

belong to red patches, and the remaining nodes belong to green patches. Sojourn time distribution parameters for ON/OFF states are shown in Table I.

In order to demonstrate the application of trained HMM in channel selection, we conduct simulations with three different channel interference conditions, and denote them as Ch1 (for channel-1), Ch2 (for channel-2), and Ch3 (for channel-3). This is equivalent to simulating three different but similar channels (for e.g., three GSM channels) experiencing different interference conditions. The interference conditions are varied from one channel simulation to another by changing the probability threshold value with which a sensor node belongs to green patch. For example, probability threshold value (pt) of 0.3 in Table III for channel Ch1 denotes that in simulations for Ch1, each node has a probability of 0.3 to belong to green patch and 0.7 to belong to red patch. Different threshold values for each channel ensures that for three channels, different number of randomly selected nodes will constitute green and red patches, thereby resulting in different interference conditions on the channels. We obtain training sequences for $\mathrm{Ch} 1, \mathrm{Ch} 2$, and $\mathrm{Ch} 3$ at the designated measurement node, and construct 2-, 3-, and 4-state trained HMMs for each of them using these sequences. We then compare the performance of these HMMs and select one HMM (2-, 3-, or 4-state) for each channel. Let us denote the selected HMMs for Ch1, Ch2, and $\mathrm{Ch} 3$ as $\mathrm{H}_{\mathrm{Ch} 1}, \mathrm{H}_{\mathrm{Ch} 2}$, and 
TABLE III

Predicted Mean CAM Values for Simulated ChanNels Based on THEIR TRAINED 2-STATE HMMS

\begin{tabular}{|l|c|c|}
\hline & $\begin{array}{l}\text { Probability } \\
\text { threshold (pt) } \\
\text { for green patch }\end{array}$ & $\begin{array}{c}\text { Predicted Mean CAM Value using } \\
\text { 2-State HMMs }\end{array}$ \\
\hline & & Mean Channel Availability Metric (CAM) \\
\hline Ch1 & 0.3 & 2.859 \\
\hline Ch2 & 0.5 & 8.288 \\
\hline Ch3 & 0.6 & 14.794 \\
\hline
\end{tabular}

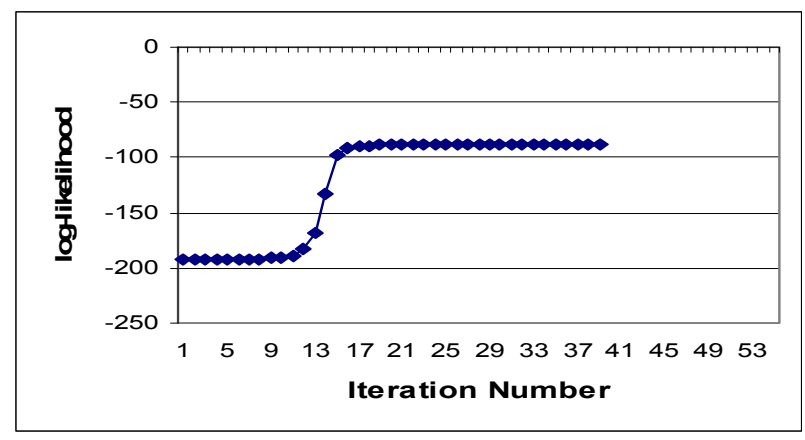

Figure 1. Log-likelihood Trend in The Training Process.

$\mathrm{H}_{\mathrm{Ch} 3}$ respectively. The measurement node uses these models to generate sequences of 600 symbols each, and calculate the channel availability metric $\left(\mathrm{CAM}_{\mathrm{i}}\right)$ for each channel using these sequences, as explained in section V. For each channel, multiple simulation runs are conducted and mean channel availability metric is obtained (with $90 \%$ confidence interval) by averaging the CAM values obtained in these runs. Mean CAM value for each channel as predicted by its corresponding HMM is given in Table III.

\section{B. Results}

We construct 2-state, 3-state and 4-state HMMs at the measurement node for all the three channels $(\mathrm{Ch} 1, \mathrm{Ch} 2$, and Ch3) by training initial models with training sequence of 300 symbols, using Baum-Welch algorithm. We initialize the model parameters (i.e. arrays A, B, and $\pi$ ) using nearly uniformly distributed values. Figure 1 shows the loglikelihood trend in the training (learning) procedure of 2-state HMM for channel $\mathrm{Ch} 2$ during one of the simulation runs. The number of iterations required to train initial HMM depend on the initial HMM parameters and training sequence symbols. Typically, the log-likelihood value increases with increasing number of iterations, till it reaches a stable maximum value at which the training terminates. Similar patterns are observed for the other HMM orders (3- and 4-states) and other channels as well.

A trained model, to be useful, must be validated on data sets (test sequences) that were not seen during training. This property is referred as generalization property [5]. Figure 2 depicts this property of trained 2-, 3-, and 4-state HMMs for channel Ch1. For a given channel and a given HMM order (number of states), we obtain a training sequence of length 300 symbols on the channel using the simulated network model. This sequence is used to train the initial HMM. Using

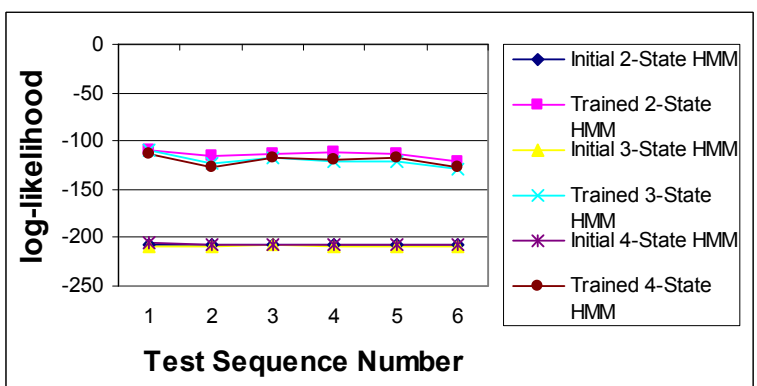

Figure 2. Log-likelihood of Test Sequences Using Initial and Trained HMMs for Channel 1.

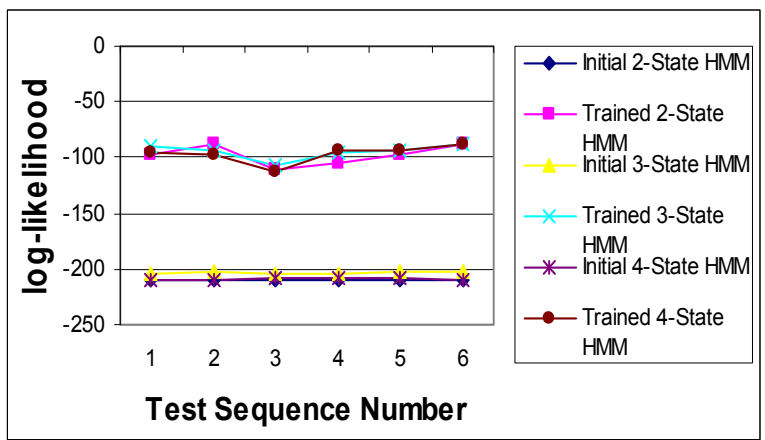

Figure 3. Log-likelihood of Test Sequences Using Initial and Trained HMMs for Channel 2.

the same simulated network model, we additionally obtain 6 different test sequences (which were not used during training phase), and compute their mean log-likelihood of occurrence (from multiple simulation runs) using trained and untrained models. As we note from Figure 2, the values of all the test sequences are considerably higher for trained models in comparison to the initial models, which indicates the significant improvement obtained using trained HMMs. Additionally, we note that the results from 2-state trained HMM are marginally better than that of 3-state and 4-state HMMs, for all the test sequences. Figure 3 and Figure 4 depicts the average log-likelihood values of the test sequences obtained using initial and trained HMMs of order 2, 3, and 4 for channel 2 and channel 3. We note that, broadly, the average values using trained models nearly overlap with each other, except for two to three sequences, where the average values obtained using 2-state trained HMM is marginally lower than that of 3-, and 4-state trained HMM. But overall, we conclude that 2-state HMM is sufficient to model all the three channels, and increasing the number of states from 2 to 3 or 4 does not bring in any significant improvement. So, for subsequent results, we use 2-state trained HMMs for Ch1, $\mathrm{Ch} 2$, and $\mathrm{Ch} 3$.

Table IV shows the channel state dynamics in steady state for channel $\mathrm{Ch} 2$. Second column of the table shows the probability of generating symbol ' 1 ' (which represents the total interference temperature exceeding the threshold) in each state (represented as (state, probability value) tuple). The third column shows the steady state probability for each state of the trained HMM (represented as SSP vector). We note that 'high interference state' $\mathrm{S} 1$ in 2-state HMM is preserved in 3-state 


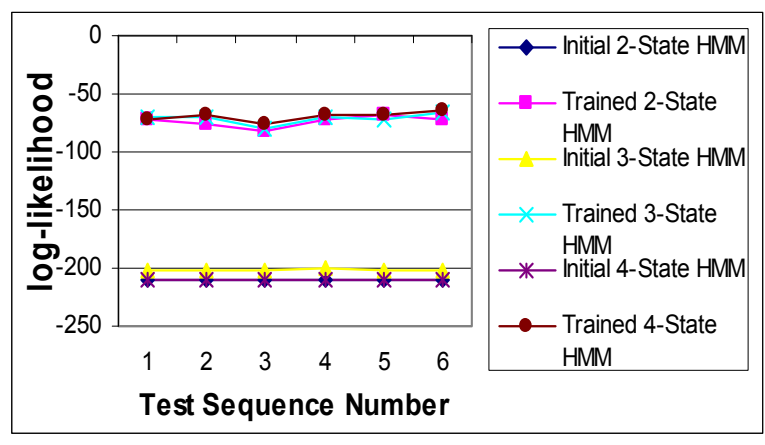

Figure 4. Log-likelihood of Test Sequences Using Initial and Trained HMMs for Channel 3 .

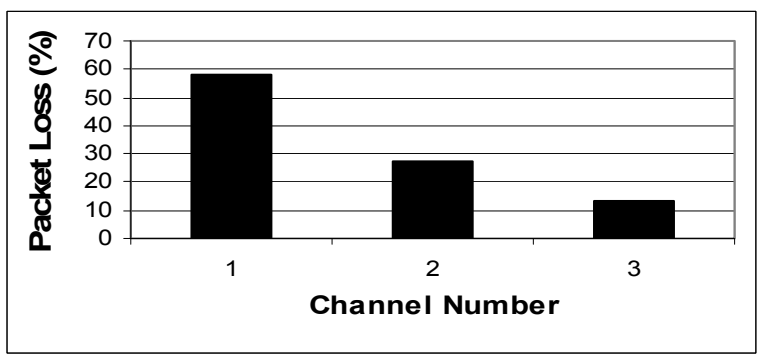

Figure 5. Observed Packet Loss (\%) on the Simulated Channels.

HMM (with same steady state probability of 0.2315 ) and is split in two states $\left(\mathrm{S}^{\mathrm{h} 1}, \mathrm{~S}^{\mathrm{h} 2}\right)$ in 4-state HMM (with steady state probability getting roughly equally divided). Similarly, the 'low interference state' S2 in 2-state HMM is divided into two states each in 3 and 4-states HMM (denoted by (S2', S2") and $\left(\mathrm{S} 2^{11}, \mathrm{~S} 2^{12}\right)$ respectively). Such pattern of splitting of states when moving from 2 states to higher number of states is observed in all the simulation runs and for other channels as well $(\mathrm{Ch} 1$ and $\mathrm{Ch} 3)$.

To demonstrate the application of trained HMM for channel selection, we consider the three channels (Ch1, Ch2, and $\mathrm{Ch} 3$ ) mentioned in the previous subsection. We generate the sequence of length 600 symbols at the measurement node using the trained 2-state $\mathrm{HMM}$ (denoted as $\mathrm{H}_{\mathrm{Ch} 1}, \mathrm{H}_{\mathrm{Ch} 2}$, and $\left.\mathrm{H}_{\mathrm{Ch} 3}\right)$ for each channel (Ch1, Ch2, and Ch3) and calculate their channel availability metric values using (3). Rightmost column of Table III shows the mean value of this metric for all the three channels. The channel availability metric predicted by trained HMM is highest for $\mathrm{Ch} 3$ and lowest for Ch1. Based on these predictions, we conclude that out of the three channels, channel-3 is most preferable and channel- 1 is least preferable for communication at the measurement node. Therefore, the measurement node should give preference to channel-3 over channel-1 and channel-2 while selecting a channel for communication. To verify the above predictions and conclusions regarding channel selection, we separately conducted multiple simulations of one hop transmission of 300 packets from a sensor node to the measurement node over the three channels and computed the mean percentage of total packet loss over each channel (with $90 \%$ confidence interval). Figure 5 shows these values for the three channels. As shown
TABLE IV

Steady State Probability of High InTERFERENCE TEMPERATURE STATES OF VARIOUS TRAINED HMMS FOR CHANNEL 2

\begin{tabular}{|l|c|c|}
\hline $\begin{array}{l}\text { HMM } \\
\text { State }\end{array}$ & Vector $\mathbf{P}$ & Vector SSP \\
\hline 2 states & $\{(\mathrm{S} 1,0.9687)$, & $\{0.2315$, \\
& $(\mathrm{S} 2,0.0127)\}$ & $0.7684\}$ \\
\hline \multirow{2}{*}{ states } & $\{(\mathrm{S} 1,0.9688)$, & $\{0.2315$, \\
& $\left.\left(\mathrm{S} 2^{\prime}, 0.00699\right),\left(\mathrm{S} 2^{\prime \prime}, 0.02075\right)\right\}$ & $0.44259,0.32585\}$ \\
\hline \multirow{2}{*}{ states } & $\left\{\left(\mathrm{S} 1^{\mathrm{h} 1}, 0.99859\right),\left(\mathrm{S} 1^{\mathrm{h} 2}, 0.83581\right)\right.$, & $\{0.17673,0.05977$, \\
& $\left(\mathrm{S} 2^{11}, 0.00861\right),\left(\mathrm{S} 2^{12}, 0.01194\right)$ & $0.39891,0.36457\}$ \\
\hline
\end{tabular}

in the figure, the percentage of lost packets observed by the measurement node on channel-3 is less than that on channel-1 and channel-2. This validates the prediction made using the trained HMMs regarding selection of Channel-3 as the preferred channel for communication.

\section{CONCLUSIONS AND FUTURE WORK}

We presented a Hidden Markov Model (HMM) for a licensed channel based on the interference temperature measured by a secondary underlay sensor network. We use this model as a generative model to predict interference dynamics in the future time slots. Results on the applicability of trained HMMs in selecting preferable channels for communication in multichannel cognitive sensor network are also presented. As a part of future work, we need to use techniques such as simulated annealing to avoid trapping of trained HMM in local maxima, and investigate theoretical methods of estimating the appropriate HMM order.

\section{REFERENCES}

[1] FCC Notice of Inquiry and Notice of Proposed Rule Making, "In the matter of establishment of an interference temperature metric to quantify and manage interference and to expand available unlicensed operation in certain fixed, mobile and satellite frequency bands," ET Docket No. 03-237, November 13, 2003.

[2] T. Clancy, "Formalizing the interference temperature model," Wiley Journal on Wireless Communications and Mobile Computing, vol. 7, (9), pp. 1077-1086, November 2007.

[3] Lawrence R. Rabiner, "A tutorial on hidden Markov models and selected applications in speech recognition," in Proc. IEEE, Vol. 77, No. 2, February 1989.

[4] Javier Garcia-Frias and Pedro M. Crespo, "Hidden Markov models for burst error characterization in indoor radio channels," IEEE Tr. On Vehicular Technology, Vol. 46, No. 4, November 1997.

[5] P. Salvo Rossi, G. Romano, F. Palmieri, and G. Iannello, "Joint end-toend loss-delay hidden Markov model for periodic UDP traffic over the Internet," IEEE Tr. On Signal Processing, Vol. 54, No. 2, February 2006.

[6] Beibei Wang, Zhu Ji, and K. J. Ray Liu, "Primary-prioritized Markov approach for dynamic spectrum access," in Proc. IEEE DySPAN 2007, Dublin, Ireland, 17 - 20 April 2007.

[7] Kyouwoong Kim, Ihsan A. Akbar, Kyung K. Bae, Jung-sun Um, Chad M. Spooner, and Jeffrey H. Reed, "Cyclostationary approaches to signal detection and classification in cognitive radio," in Proc. IEEE DySPAN 2007, Dublin, Ireland, 17 - 20 April 2007.

[8] Joe Bater, Hwee-Pink Tan, K. N. Brown, and Linda Doyle, "Modeling Interference Temperature Constraints for Spectrum Access in Cognitive Radio Networks," in Proc. IEEE CogNet Workshop, June 2007.

[9] OPNET Technologies, Inc. (www.opnet.com) 